\title{
Comparison of $T$ and NK lymphocyte subsets between human endometrial tissue and peripheral blood
}

\author{
VIKTOR P. CHERNYSHOVI, BORIS V. DONS'KOI', IRYNA O. SUDOMA', \\ YANA O. GONCHAROVA ${ }^{2}$ \\ ${ }^{1}$ Laboratory of Immunology, Institute of Pediatrics, Obstetrics and Gynecology, National Academy of Medical Sciences of Ukraine, \\ Kyiv, Ukraine \\ ${ }^{2}$ Reproductive Medicine Clinic "Nadiya”, Kyiv, Ukraine
}

\begin{abstract}
Immune profiles in endometrium may be changed in patients with IVF failure and its possible correlations with immune parameters in peripheral blood are important for the diagnostic approach. Such correlations in healthy women are unknown and have been studied in the present research. The expression of CD56, CD158a, HLA DR, CD69 in T lymphocytes, CD4 T lymphocytes, CD8+ T lymphocytes and NK cells were studied by flow-cytometry in endometrium and peripheral blood in healthy 24 donors of oocytes aged 25-32 years. Levels of T lymphocyte and Thelper cells were lower in endometrium and no differences in CD8 T lymphocytes were registered between endometrium and peripheral blood. The expression of HLA DR and especially CD69 was higher in CD3, CD4, CD8 T cells in endometrium in comparison with peripheral blood. The endometrium lymphocyte population was enriched by NK cells that were generally CD56++ with a higher expression of HLA DR and almost in total were CD69 positive. Strong positive correlations of CD8 expression in NK cells $(r=0.6478, p<0.001)$ and HLA DR expression in CD8 T cells $(r=0.6107, p<0.01)$ between peripheral blood and endometrium were registered in fertile women. The endometrial CD56 expression in CD8+T cells negatively correlated with endometrial CD8 expression in NK cells $(r=-0.5252, p<0.01)$ which possibly reflected a suppressive and regulating mechanism in the endometrium. CD8+NK cells and HLA DR+CD8 T cells in endometrium were related to the same subsets in peripheral blood.
\end{abstract}

Key words: endometrium, peripheral blood, immune profiles.

(Centr Eur J Immunol 2019; 44 (3): 316-321)

\section{Introduction}

Endometrial immune profiles are registered as dysregulated in most of the patients with recurrent implantation failures compared to controls [1]. Some authors confirmed the association of uterine NK cells and infertility [2-4], while others found no association [5, 6]. CD16+: CD56+ and CD56+: NKp46+ cell ratios in the endometrium were suggested to be important for recurrent pregnancy losses (RPL) and infertility [7]. In patients with recurrent miscarriages, no correlation was found between peripheral and uterine NK cells but an association between peripheral lymphocytes (CD45+CD3-DR+ and CD45+CD3+C$\mathrm{D} 8+\mathrm{DR}+$ ) and uterine NK cells was confirmed and it was supposed that T, B and NK lymphocytes provided cytokines for the differentiation of uterus NK cells [8].

In women with IVF failure and RPL various immune deviations were registered in peripheral blood including increased levels and elevated activity of NK cells [9-13], predominance of Th1 immune response, elevated expression of activation markers in T cell subsets [14], imbalance of KIR receptors expression in NK cells [15-17], elevated number of NKT cells [18]. It was shown that a certain elevated level of NK cells and NK cytotoxicity might have potentially an adverse effect for reproduction [13, 19]. Some parameters that were out of favorable limits (elevated expression of CD56, CD158a in T lymphocytes, decreased levels of CD4 T lymphocytes, up-regulated expression of HLA DR in CD8+ T cells and NK cells, elevated number of NK cells and increased NK cytotoxicity, increased and decreased expression of CD158a and CD8 in NK cells) were considered in our study to be immune deviations (ID) potentially predictive for IVF failure [17]. A combination of ID may predict implantation failure to a greater degree than isolated ID. Multiple immune devi-

Correspondence: Prof. Viktor P. Chernyshov, MD, PhD, DSc, Laboratory of Immunology, Institute of Pediatrics, Obstetrics and Gynecology, National Academy of Medical Sciences of Ukraine, 8 Platon Maiboroda St., 04050, Kyiv, Ukraine, e-mail: chernyshov@ukr.net Submitted: 19.09.2017; Accepted: 31.10.2017 
ations form an unfavorable "immune phenotype" for implantation and pregnancy development [20].

The association of immune population in peripheral blood with a local endometrium condition is the main diagnostic problem in reproductive immunology that is still a matter of debate. Until now the question of lymphocyte subsets association between endometrium and peripheral blood is not clear enough even in healthy women.

In this study we aimed to make the first step to compare the levels of T and NK lymphocyte subsets in endometrium and peripheral blood in donors of oocytes (healthy, fertile women).

\section{Material and methods}

The study was conducted as a prospective study. Twenty five donors of oocytes aged 25-30 years who agreed to endometrial biopsy were recruited from an in-vitro fertilization (IVF) clinic - Reproductive Medicine Clinic "Nadiya", Kyiv, Ukraine. The local ethical committee's approval in accordance with the Helsinki Declaration of 1975 on human experimentation and patient's informed consent were obtained.

A long luteal protocol using GnRH-a (diphereline $3.75 \mathrm{mg}$ ) and recombinant gonadotropin (Gonal- F) was applied for controlled ovarian hyperstimulation.

Endometrial samples were obtained by pipelle biopsy (catheter biopsy) immediately after oocytes retrieval, 34-36 hours after hCG trigger (Ovitrel $250 \mathrm{mg}$ ). Blood sampling for immunological studies was taken on the same day 25-30 min prior to endometrial biopsy.

\section{Peripheral blood lymphocytes study}

Lymphocyte subsets were studied by three-color flow cytometry in whole blood after staining with monoclonal antibodies conjugated with fluorochromes FITC, PE, PE-Cy 5 (BD Biosciences, USA) in the following combinations: CD158aFITC/CD56PE/CD3PE Cy5, CD8FITC/CD56PE/CD3PE Cy5, HLA DR FITC/CD56PE/ CD3PE Cy5, HLA DR FITC/CD8PE/CD3PE Cy5, CD69FITC/CD56PE/CD3PE Cy5, CD62L/CD56PE/ CD3PE Cy5. Blood samples were analyzed with a FACScan flow cytometer (BD Biosciences, USA). For determining the lymphocyte gate, the whole blood samples were incubated with mAbs of LeucoGATE CD45FITC/CD14PE kit to permit discrimination of lymphocytes from granulocytes, monocytes, debris and unlysed or nucleated red blood cells. In addition, the whole blood samples were incubated with SimulTEST control (IgG1-FITC + IgG2-PE) as a background control. We referred to the recommendations of BD Biosciences for normal ranges of the main lymphocyte subsets in peripheral blood [21].

\section{Endometrial lymphocytes study}

Endometrial tissue samples were gently washed three times in phosphate-buffered saline (PBS) to release from blood contamination (in some samples).

$1 \mathrm{ml}$ pipette tip was cut at the edge so that the diameter of the hole was $4 \mathrm{~mm}$ and endometrial tissue was washed 10-15 times by pipetting in PBS in order to obtain free endometrial cells in PBS solution. The next pipette tip had a hole diameter of 2-3 $\mathrm{mm}$ and tissue pieces were pipetted again. At the last step of pipetting, the tip hole was 1-2 $\mathrm{mm}$ and pieces were pipetted without resistance, i.e. every next step was done with a smaller diameter of the tip hole. Endometrial tissue was disaggregated and made flakes-shaped. $7 \mathrm{ml}$ of PBS $\left(+4^{\circ} \mathrm{C}\right)$ were added, mixed with the obtained suspension and remained for 3-5 min for sedimentation of big aggregates. Cellular supernatant was carefully layered onto $3 \mathrm{ml}$ of Histopaque- 1077 in $15 \mathrm{ml}$ conical centrifuge tube and centrifuged at $400 \mathrm{~g}$ for $30 \mathrm{~min}$ at room temperature. After centrifugation, the upper layer was carefully aspirated and this opaque interface was carefully transferred into a clean conical centrifuge tube. The cells were washed twice by $10 \mathrm{ml}$ of PBS. Cells pellet was resuspended in $0.5 \mathrm{ml}$ of PBS with $10 \%$ of fetal bovine serum. Final cell suspension was analyzed by flow cytometry as shown in Peripheral blood lymphocytes study (upper).

Statistical analysis was performed using InStat for Windows (version 3.0, Graph Pad Software Inc., San Diego, USA). The significance of differences was calculated by the Mann-Whitney test (unpaired, non-parametric, two-tailed), with $95 \%$ confidence interval (CI). Correlations by Pearson and Spearman were used too. Differences with $p<0.05$ were considered statistically significant.

\section{Results}

More than one million of mononuclear lymphocytes (without erythrocyte and debris contamination) were isolated out of each 24 samples of endometrium, one sample with low cell numbers was RBC and debris contaminated and was not analyzed. Endometrium lymphocyte population was enriched by NK cells that were generally CD56++ and almost totally CD69+ which confirmed absence of blood lymphocytes contamination (Fig. 1).

\section{Comparison of blood and endometrium}

Percentage levels of T and NK lymphocyte subsets in endometrium and peripheral blood of healthy fertile women were compared in samples that were received on the same day. The levels of T lymphocytes (CD3+) were significantly lower in endometrium compared to peripheral blood. The expression of HLA DR in T lymphocytes was higher in endometrium. Moreover, in endometrium a very high expression of CD69 was registered in CD3+ and NK 

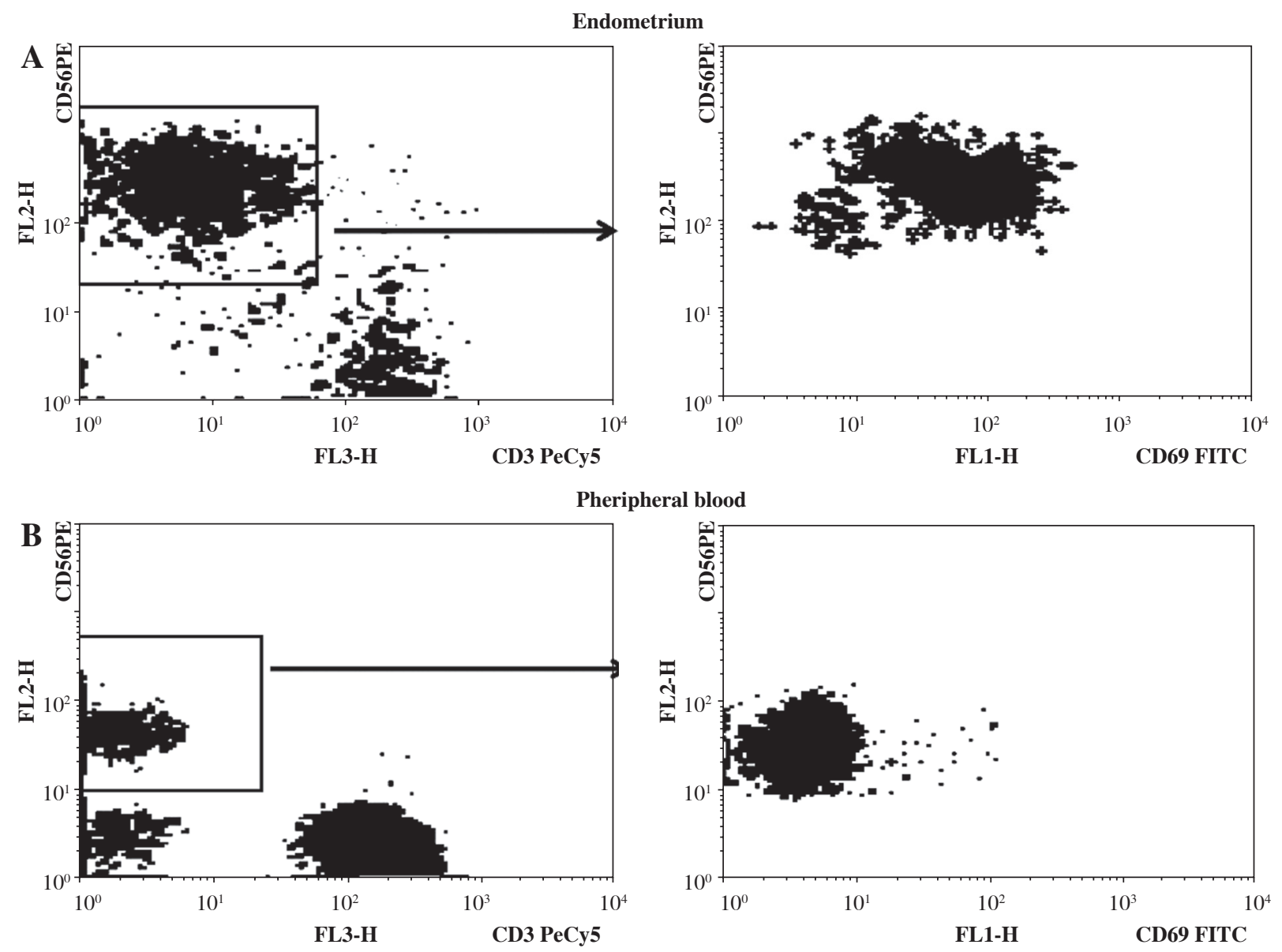

Fig. 1. Differential CD56 (left) and CD69 (right) expression in endometrium (A) and peripheral blood (B) and lymphocytes

lymphocytes in comparison to a very low expression of CD69 in CD3+ and NK lymphocytes of peripheral blood. The differences in the expression of CD56 and CD158a in CD3+ lymphocytes of endometrium and peripheral blood were not significant (Table 1).

The levels of $\mathrm{T}$ helper cells (CD3+CD4+) in endometrium were lower compared to peripheral blood. But the expression of HLA DR in CD3+CD4+ was higher in endometrium. There were no differences in $\mathrm{CD} 3+\mathrm{CD} 8+\mathrm{T}$ lymphocytes in the endometrium and peripheral blood. But the expression of HLA DR in CD3+CD8+ was higher in the endometrium. The level of CD3+CD8+CD56+ in endometrium was higher than in peripheral blood (Table 1).

The level of NK cells (CD56+CD3-) was higher in endometrium compared to peripheral blood. No difference in endometrium and peripheral blood was noted as for the levels of expression of CD158a (CD56+CD3-CD158a+). Higher activation of NK cells was observed in endometrium. The expression of HLA DR in NK cells (HLA DR+ [\%] in CD56+CD3-) and especially CD69 (CD69+ [\%] in CD56+CD3-) was higher in endometrium than in peripheral blood. The expression of CD8 in NK cells (CD8 [\%] in CD56+CD3-) in endometrium was lower than in peripheral blood. The levels of NK cells bright (CD56++CD3-) were extremely higher in endometrium than in peripheral blood (Table 1).

\section{Correlations between blood and endometrium}

No significant correlations were observed between blood and endometrium NK and T cell levels. But an extremely significant positive correlation was registered in levels of CD8+ in NK cells (CD8+ [\%] in CD56+CD3-) between endometrium and peripheral blood (Fig. 2). In addition, a correlation was noted between peripheral blood and endometrium expression of HLA DR (\%) in CD3+CD8+T lymphocytes (Fig. 3).

It was interesting that endometrial CD56 expression in CD8+ T cells negatively correlated with endometrial CD8 expression in NK cells (Fig. 4). There was no such correlation in peripheral blood.

\section{Discussion}

The present study confirms that endometrium presents a wide spectrum of T and NK lymphocyte subsets. Endo- 
Table 1. T and NK lymphocyte subsets $(\%)$ of peripheral blood and endometrium in fertile women $(n=24)$

\begin{tabular}{lcc}
\hline T and NK lymphocyte subsets $(\%)$ & Peripheral blood, median (95\% CI) & Endometrium, median $(\mathbf{9 5 \%}$ CI) \\
\hline CD3+ (T cells) \% & $75.0(73.1-77.1)$ & $60.1(54.1-61.3)^{* * *}$ \\
\hline$\%$ HLA DR+ in T cells & $11.9(10.3-14.2)$ & $43.6(40.6-48.9)^{* * * *}$ \\
\hline$\%$ CD69+ in T cells & $3.2(2.7-4.5)$ & $89.6(80.3-92.1)^{* * *}$ \\
\hline$\%$ CD56+ in T cells & $7.9(5.5-15.3)$ & $8.4(7.7-13.4)$ \\
\hline$\%$ CD158a+ in T cells & $2.8(2.5-6.0)$ & $3.6(3.3-10.3)$ \\
\hline$\%$ CD4-CD8- in T cells & $5.4(4.1-6.9)$ & $3.2(3.1-8.3)$ \\
\hline CD3+CD4+ (T helper cells) \% & $44.2(42.3-47.1)$ & $26.9(22.6-29.3)^{* * *}$ \\
\hline$\%$ HLA DR+ in T helper cells & $6.6(5.7-7.7)$ & $30.9(27.3-34.8)^{* * *}$ \\
\hline$\%$ CD56+ in T helper cells & $1.4(-3.1-15.6)$ & $4.3(3.5-6.4)^{* * *}$ \\
\hline CD3+CD8+ (T cytotoxic cells) \% & $26.2(23.4-27.4)$ & $26.2(24.6-30.6)$ \\
\hline$\%$ HLA DR+ in T cytotoxic cells & $20.5(18.3-25.7)$ & $49.8(40.2-51.1)^{* * * *}$ \\
\hline$\%$ CD56+ in T cytotoxic cells & $7.8(6.1-11.3)$ & $12.6(9.6-14.2)^{*}$ \\
\hline CD56+CD3- (NK cells) \% & $9.7(8.9-12.5)$ & $29.0(25.9-35.5)^{* * *}$ \\
\hline$\%$ CD158a+ in NK cells & $20.2(19.0-32.8)$ & $17.7(15.0-22.9)$ \\
\hline$\%$ HLA DR+ in NK cells & $11.6(10.5-17.2)$ & $23.4(19.4-27.5)^{* * *}$ \\
\hline$\%$ CD69+ in NK cells & $4.5(4.0-5.7)$ & $91.1(87.1-93.1)^{* * * *}$ \\
\hline$\%$ CD8+ in NK cells & $42.3(37.2-48.2)$ & $10.9(13.5-25.8)^{* * * *}$ \\
\hline$\%$ CD56++ bright in NK cells & $8.1(7.5-12.5)$ & $74.4(68.2-80.1)^{* * * *}$ \\
\hline
\end{tabular}

metrial T lymphocytes levels were lower in comparison to blood lymphocytes but extremely activated which was confirmed by a high expression of HLA DR and especially by an extreme expression of the early marker of activation CD69 in CD3+ cells in endometrium. Our method of preparation of endometrial lymphocytes for analysis was suitable for the study of subsets. We observed a positive

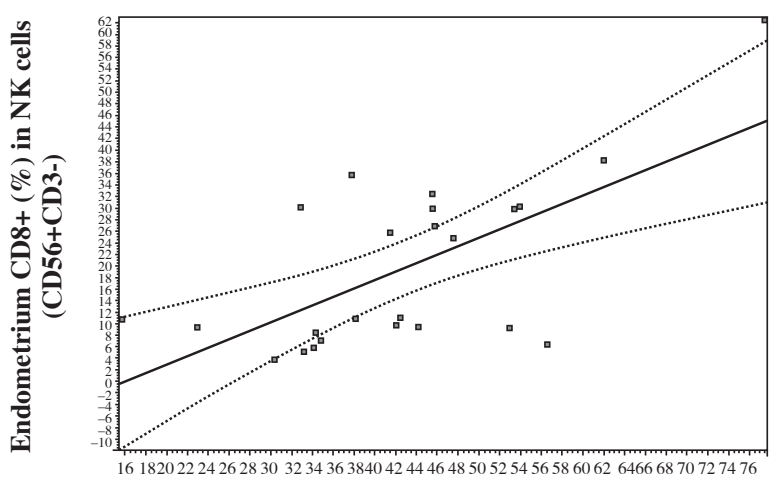

Peripheral blood CD8+ $(\%)$ in NK cells (CD56+CD3-)

Fig. 2. Correlation in the levels of CD8+ (\%) in NK lymphocytes (CD56+CD3-) between peripheral blood and endometrium (Pearson correlation $r=0.6478, p<0.001$, 95\% CI: 0.3307-0.8334; Spearman correlation $r_{s}=0.498$, $p<0.01,95 \%$ CI: 0.1058 to 0.7561 ) correlation between peripheral blood and endometrium in HLA DR+ cytotoxic T lymphocytes (HLA DR+ [\%] in $\mathrm{CD} 3+\mathrm{CD} 8+$ ). Earlier we confirmed that this subset level elevation in blood was associated with IVF failure [20]. Recently, it has been indicated that CD8+ HLA DR+ T cells constitute to a natural subset of Tregs and may represent a separate lineage of $\mathrm{CD} 8+\mathrm{T}$ cells. A suppressive

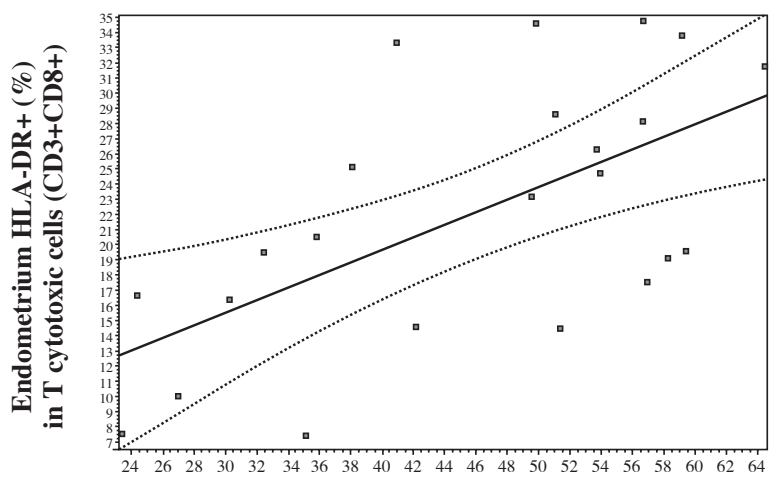

Peripheral blood HLA-DR+ $(\%)$ in T cytotoxic cells (CD3+CD8+)

Fig. 3. Correlation in the levels of HLA DR+ (\%) in CD8+ $\mathrm{T}$ lymphocytes between peripheral blood and endometrium (Pearson correlation $r=0.6107, p<0.01$; 95\% CI: 0.26510.8172 ; Spearman correlation $r_{s}=0.5337, p<0.01,95 \%$ CI: 0.1430 to 0.7805 ) 


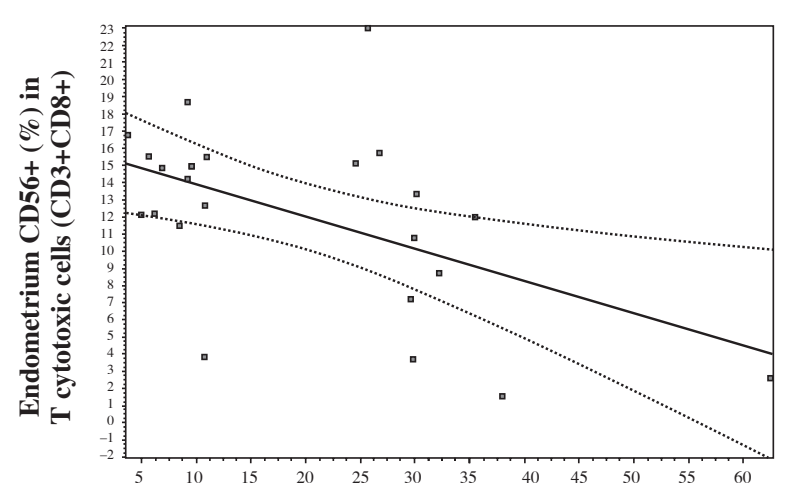

Endometrium CD8+ $(\%)$ in NK cells $(\mathrm{CD56}+\mathrm{CD3}-)$

Fig. 4. Correlation between the levels of $\mathrm{CD} 8+(\%)$ in NK lymphocytes (CD56+CD3-) and CD56+(\%) in CD8+ T lymphocytes (CD8+CD3+) in endometrium (Pearson correlation $r=-0.5252, p<0.01 ; 95 \% \mathrm{CI}:-0.7663$ to -0.1544 ; Spearman correlation $r_{s}=-0.5120, p=0.0105$, 95\% CI: -0.7640 to 0.1243 )

effect of these cells appears to be cell-to-cell contact dependent and may involve CTLA-4 signaling between neighboring $\mathrm{T}$ cells. These $\mathrm{T}$ cells can be expanded in vitro and may exhibit a suppressive capacity similar to that observed in vivo in CD8+ HLA DR+ T cells [22]. A correlation in activated $\mathrm{T}$ cells between peripheral blood and endometrium, which was confirmed in our study, may reflect some joint regulatory mechanisms, including $\mathrm{T}$ cells in peripheral blood and endometrium.

According to our data, in controlled ovarian hyperstimulation cycles, the level of NK cells in endometrium 34-36 hours after hCG trigger injection was significantly higher than in peripheral blood (about $30 \%$ ). It corresponds to the data of other researchers who observed a similar pattern when studying the correlation of NK cells in peripheral blood and secretory endometrium [23]. Endometrium NK cells in our study were dramatically activated and had a higher expression of HLA DR and especially CD69 in CD56+CD3- cells compared to the same subsets in peripheral blood. We have previously shown that CD69 expression after K562 stimulation of blood NK cells reflects their function and is associated with reproductive failures [24].

No difference in the levels of CD158a expression in NK cells but a lower expression of CD8 was found in endometrial NK cells. Extremely high levels of CD56++bright NK cells (CD56++CD3-) are inherent to endometrium and this was confirmed in our study. We observed no correlation in the whole subset of NK cells between peripheral blood and endometrium in healthy donors of oocytes which corresponds to other publications $[8,25]$. But in the present study we have shown a strong correlation in CD8 expression in NK cells and HLA DR expression in cytotoxic T lymphocytes between peripheral blood and endometrium. Previously we have demonstrated that CD8 accentuated expression on blood NK cells was associated with reproductive failures $[26,27]$.

CD8+ NK cells (CD8+ [\%] in CD56+CD3-) are highly functional; they exhibit a more functional profile (as detected by cytokine production and degranulation) and are inversely associated with HIV-related disease markers and linked with delayed disease progression [28]; they are also more radio-resistant [29] and more cytotoxic which facilitates tumor killing [30].

According to our results, a CD8 positive NK cells level in endometrium of healthy fertile women is twice lower than in peripheral blood. However, this level may possibly change in women with reproductive problems.

A lymphocyte lineage which is referred to as NKT cells expresses both markers of NK cells and T lymphocytes. NKT cells play a key role in the regulation of different types of immune responses; contribute to the protection from tumor growth and metastasis, from different intracellular infections and from development of autoimmune diseases. NKT cells are of vital importance in the induction of antitumor immune response due to their production of IFN- $\gamma$ which activates NK cells, CD8+ T lymphocytes and macrophages [31]. Our results showed that there is a negative correlation between subsets: CD56+ (\%) in CD8+ T cells and CD8+ (\%) in NK cells in endometrium but not in peripheral blood. Perhaps, it is related to the local feature - a more expressive and specific mechanism of suppression and regulation in the endometrium zone. Both these subsets are related to the suppression and possibly the negative correlation reflects a more effective permanent suppressive and regulating complex mechanism including compensation when these subsets are in normal values. Obviously, there is such competition between NK "T-like function" and T "NK-like mechanisms". Previously, we have found that both these subsets in abnormal levels are related with IVF failure [20, 27]. We have not found differences in KIR (CD158a) expression in T cells and NK cells between peripheral blood and endometrium and it will be interesting in the future to study KIR phenotype of NK cells and to do more characterization of functional properties of NK cells and T cells such as degranulation and/or cytokine production.

\section{Conclusions}

Our study confirmed that there is no association between the main subsets of T and NK cells of peripheral blood and endometrium in healthy fertile women. However, our first step of study shows that such association exists for some separate subsets - HLA DR+ cytotoxic T lymphocytes (HLA DR+ [\%] in CD3+CD8+) and subsets pattern of NK population bearing CD8 (CD8+ [\%] in CD56+CD3-), which may reflect some regulatory mechanisms. In future studies, it is important to control such correlations in the period of "implantation window" and 
adding of these parameters to peripheral blood tests may give additional information about local immune conditions in patients with IVF failure and RPL.

The authors declare no conflict of interest.

\section{References}

1. Lédée N, Prat-Ellenberg L, Chevrier L, et al. (2017): Uterine immune profiling for increasing live birth rate: A one-to-one matched cohort study. J Reprod Immunol 119: 23-30.

2. Quenby S, Bates M, Doig T, et al. (1999): Pre-implantation endometrial leukocytes in women with recurrent miscarriage. Hum Reprod 14: 2386-2391.

3. Clifford K, Flanagan AM, Regan L (1999): Endometrial CD56+ natural killer cells in women with recurrent miscarriage: a histomorphometric study. Hum Reprod 14: 2727-2730.

4. Lédée-Bataille N, Bonnet-Chea K, Hosny G, et al. (2005): Role of the endometrial tripod interleukin-18, -15 , and -12 in inadequate uterine receptivity in patients with a history of repeated in vitro fertilization-embryo transfer failure. Fertil Steril 83: 598-605.

5. Lachapelle MH, Miron P, Hemmings R, Roy DC (1996): Endometrial T, B, and NK cells in patients with recurrent spontaneous abortion. Altered profile and pregnancy outcome. J Immunol 156: 4027-4034.

6. Matteo MG, Greco P, Rosenberg P, et al. (2007): Normal percentage of CD56 bright natural killer cells in young patients with a history of repeated unexplained implantation failure after in vitro fertilization cycles. Fertil Steril 88: 990-993.

7. Giuliani E, Parkin KL, Lessey BA, et al. (2014): Characterization of uterine NK cells in women with infertility or recurrent pregnancy loss and associated endometriosis. Am J Reprod Immunol 72: 262-269.

8. Kuon RJ, Vomstein K, Weber M, et al. (2017): The "killer cell story" in recurrent miscarriage: Association between activated peripheral lymphocytes and uterine natural killer cells. J Reprod Immunol 119: 9-14.

9. Kwak JY, Beaman KD, Gilman-Sachs A, et al. (1995): Up-regulated expression of CD56+, CD56+/CD16+, and CD19+ cells in peripheral blood lymphocytes in pregnant women with recurrent pregnancy losses. Am J Reprod Immunol 34: 93-99.

10. Emmer PM, Nelen WI, Steegers EA, et al. (2000): Peripheral natural killer cytotoxicity and CD56(pos) CD16(pos) cells increase during early pregnancy in women with a history of recurrent spontaneous abortion. Hum Reprod 15: 1163-1169.

11. Matsubayashi H, Hosaka T, Sugiyama Y, et al. (2001): Increased natural killer-cell activity is associated with infertile women. Am J Reprod Immunol 46: 318-322.

12. Ntrivals EI, Kwak-Kim JYH, Gilman-Sachs A, et al. (2001): Status of peripheral blood natural killer cells in women with recurrent spontaneous abortions and infertility of unknown etiology. Hum Reprod 16: 855-861.

13. Karami N, Boroujerdnia MG, Nikbakht R, Khodadadi A (2012): Enhancement of peripheral blood CD56(dim) cell and NK cell cytotoxicity in women with recurrent spontaneous abortion or in vitro fertilization failure. J Reprod Immunol 95: 87-92.

14. Kwak-Kim JYH, Chung-Bang HS, Ng SC, et al. (2003): Increased $\mathrm{T}$ helper 1 cytokines responses by circulated $\mathrm{T}$ cells are present in women with recurrent pregnancy losses and in infertile women with implantation failures after IVF. Hum Reprod 18: 767-773.

15. Coulam CB, Roussev RG (2003): Correlation of NK cell activation and inhibition markers with NK cytotoxicity among women experiencing immunologic implantation failure after in vitro fertilization and embrio transfer. J Assist Reprod Genet 20: $58-62$.

16. Ntrivals EI, Bowser CR, Kwak-Kim J, et al. (2005): Expression of killer immunoglobulin-like receptors on peripheral blood NK cell subsets of women with recurrent spontaneous abortions or implantation failures. Am J Reprod Immunol 53: 215-221.

17. Chernyshov VP, Sudoma IO, Dons'koi BV, et al. (2010): Elevated NK cell cytotoxicity, CD158a expression in NK cells and activated T lymphocytes in peripheral blood of women with IVF failures. Am J Reprod Immunol 64: 58-67.

18. Heilmann L, Schorsch M, Hahn T (2010): CD3-CD56+CD16+ natural killer cells and improvement of pregnancy outcome in IVF/ICSI failure after additional IVIG-treatment. Am J Reprod Immunol 63: 263-265.

19. Beer AE, Kwak JY, Ruiz JE (1996): Immunophenotypic profiles of peripheral blood lymphocytes in women with recurrent pregnancy losses and in infertile women with multiple failed in vitro fertilization cycles. Am J Reprod Immunol 35: 376-382.

20. Chernyshov VP, Dons'koi BV, Sudoma IO, Goncharova YO (2014): Favorable immune phenotype predicts successful implantation and pregnancy. Immunol Lett 162: 217-221.

21. Comans-Bitter WM, Groot R, Beemed R, et al. (1997): Reference values for lymphocyte subpopulations. J Pediatr 130: 388-393.

22. Arruvito L, Payaslián F, Baz P, et al. (2014): Identification and clinical relevance of naturally occurring human $\mathrm{CD} 8+\mathrm{H}-$ LA-DR+ regulatory T cells. J Immunol 193: 4469-4476.

23. Moffett A, Regan L, Braude P (2004): Natural killer cells, miscarriage, and infertility. BMJ 329: 1283-1285.

24. Dons'koi BV, Chernyshov VP, Sirenko VY, et al. (2014): Peripheral blood natural killer cells activation status determined by CD69 upregulation predicts implantation outcome in IVF. Immunobiology 219: 167-171.

25. Zhang Y, Kwak-Kim J, Huang D, et al. (2014): Cyclic changes and relationship between peripheral and endometrial NK cells from women with repeated failure after artificial insemination by donor sperm. Am J Reprod Immunol 71: 44-54.

26. Dons'koi BV (2015): Accentuated hypo- and hyper-NK lymphocyte CD8 expression is a marker of NK subsets' misbalance and is predictive for reproductive failures. Immunobiology 220: 649-655.

27. Chernyshov VP, Dons'koi BV, Sudoma IO, Goncharova YO (2016): Multiple immune deviations predictive for IVF failure as possible markers for IVIG therapy. Immunol Lett 176: 44-50.

28. Ahmad F, Hong HS, Jäckel M, et al. (2014): High frequencies of polyfunctional CD8+ NK cells in chronic HIV-1 infection are associated with slower disease progression. J Virol 88: $12397-12408$

29. Vokurková D, Vávrová J, Šinkora J, et al. (2010): Radiosensitivity of CD3-CD8+CD56+ NK cells. Radiat Meas 45: 1020-1023.

30. Suck G, Chu S, Niam M, et al. (2007): Highly cytotoxic CD56+CD8+ NK cells as potential novel candidates for cancer cellular therapy. Clin Cancer Res 13: B13.

31. Godfrey DI, Stankovic S, Baxter AG (2010): Raising the NKT cell family. Nat Immunol 11: 197-201. 